\title{
Micro-mechanical (granular) mixtures for environmental safety technologies
}

\author{
Oleg Gerasymov ${ }^{1, *}$, Andrii Spivak ${ }^{1}$, Iryna Andrianova ${ }^{1}$, Liudmyla Sidletska ${ }^{1}$, Vladyslav Kuryatnikov ${ }^{1}$, and Andrii Kilian ${ }^{1}$ \\ ${ }^{1}$ Odessa State Environmental University, Department of the General and Theoretical Physics, 15 Lvivska St., Odesa, 65016, Ukraine
}

\begin{abstract}
One of the most significant problems that belong to effective use of granular materials GM (for example, in the construction, pharmacological, chemical, agricultural and some other industries, as well as in the technologies of screening against internal radiation) is the difficulty of ensuring their maximum compaction in order to increase the efficiency of their practical application. In this way, we study of the properties of binary granular systems, the dynamics of their compaction and the impact on this process of the ratio of component sizes and partial parameters in order to provide an innovative step in the development of appropriate technologies. To this end, we propose to use the apparatus of Kirkwood-Buff theory in combination with model equations of state, like Carnahan-Starling, together with relevant phenomenological information, which obtained from direct observations. We report theoretically the fundamental possibility of increasing the degree and speed of packaging and at first describe substantiate empirical data in the full range of values of the volume (or molar) fraction.
\end{abstract}

\section{Introduction}

Among the typical problems of environmental protection technologies, we deal with multicomponent, polydisperse, multiparameter systems that perform an insulating, protective function. The understanding of the principles due to which the basic parameters of such systems are formed is based on theoretical models that allow to treat the measurement data in terms of quantities that characterize the individual pure components (reference data).

The construction of such a models is a very difficult task and, first, requires phenomenological information from several alternative sources. And secondly, the definition of the partial parameters themselves, or rather their relationship to the real mixture, as well as the definition of its ideal (mixed) states is quite controversial and only partially theoretically argumented procedure.

In this paper, being based on the apparatus of Kirkwood-Buff theory, model Carnahan-Starling equation of state and relevant experimental data we study theoretically the macroscopic parameters of bidispersed micro-mechanical mixture in order to build algorithm for practical describing and parameterizing their physical and mechanical characteristics in terms of its partial properties depending from the volume (or molar) fraction of one of the component.

We offer a constructed approach to use in practice to parameterize the properties of two-component granular materials in order to optimize the technological processes in which they are used (in particular, in the relevant technologies of granular protective screens).

Several theoretical models and approaches will be used to consider the outlined problem. Namely: theoretical models of granular bi-component mixtures, methods of theoretical statistical physics, in particular Kirkwood-Buff theory, model equation of state of the mixture of solid balls (Carnahan-Starling equation), phenomenological information about the dynamics of compaction of the binary granular mixtures.

Using Kirkwood-Buff theory, model relations for mixtures of solid balls and phenomenological data on the compaction of granular materials, we came out with a working formula for describing the macroscopic properties of binary granular systems in terms on the partial parameters of its components, such as their volume or molar fractions.

The obtained data in particular confirm the influence of multi-dispersion on the dynamics of compaction (namely, we outline the role which play the absolute value and rate of packaging).Therefore we report the possibility of the mixture under the influence of external perturbation to change the local structure of packaging and its parameters (in particular, degree and velocity of compaction).

The use of materials that are in granular (micromechanical) states is inherent in many industries and technologies. For example, granular materials make up the predominant percentage of products that are produced, consumed or used in certain stages of construction, food, mining, chemical and pharmaceutical, metallurgical and some other industries. Special sector of application of such a materials is environmental safety technologies.

The variety of applications of such materials requires the ability to reliably parameterize their macroscopic properties, taking into account the external influences that arise when manipulating them. The relationship between the macroscopic properties of the granular material (for example, ordering in the form of

Corresponding author: gerasymovoleg@gmail.com 
crystallization) and its macroscopic parameters, which purely determine its ability to maintain normal modes and provide the necessary manipulation also plays an important role .Such as: delay or transmit radiation of various natures, keep loading due to the arched effect, or avoid aggregation when mixing.

One of the examples in which there is a significant need for algorithms for directed manipulation of macroscopic parameters by external influences is designs that use protective modules containing a granular substance. Such "granular screens" can potentially perform their regular functions quite reliably and at the same time have obvious advantages over monolithic systems. So, in particular, they: avoid radiation embrittlement; easy to manipulate; involve compaction and loosening; easy to replace and to transport; economic (in the sense of their production and operation).

In general, problems about mixtures occupy a prominent place in engineering models for forecasting the state of the environment. For example, work [1] is devoted to forecasting sediment transport and river morphodynamics (which is a difficult task in rivers with a gravel bed, where sediment mixtures include a particle with different sizes). The authors of [1] show that the problem of binary packing is important for the correct calculation of the porosity of the gravel layer. In practice, their approach can be combined with calculations of relevant dynamic models of sludge transport to predict morphodynamics in rivers with gravel bottom.

Theoretical models of granular materials that would allow describing and predicting their properties are developed quite carefully for the cases of specific conditions of their creation and use, in particular the dimension, density of packaging, the nature of external influences. Unfortunately, there is still no universal theory of granular materials that takes into account all the observed properties. But in many specific cases it is possible to offer more or less adequate models of the description of their behavior.

Materials used, for example, in nuclear reactors, are affected by intense fluxes of ionizing radiation, mainly fast neutrons, which are products of fission of nuclear fuel. Neutrons, interacting with matter, transfer their energy to atoms through elastic collisions, and also serve as a source of formation of fast charged particles (electrons, protons, $\alpha$-particles). The expected result of these processes is the destruction of the ordered arrangement of atoms in the crystal lattice of the metal and the formation of foreign atoms, such as helium and hydrogen. The set of radiation changes in the structure of the material is called radiation damage (or radiation embrittlement) [2].

A characteristic phenomenon that can accompany radiation damage of the material is the shift of atoms from the nodes of the crystal lattice and the formation of point defects - vacancies and interstitial atoms. A characteristic feature of fast neutron irradiation is the occurrence of cascades of atomic collisions in the irradiated material, which lead to the formation of microscopic areas of structural damage with a high concentration of point defects. Under the influence of temperature, the initial state of damage changes as a result of thermally activated migration of point defects, which is accompanied by their mutual recombination, annihilation in runoff, formation or dissociation of clusters.

In the radiation industry, it is known that the brittle destruction of metals in practice occurs when the strength criterion of 500-600 MPa. It was found [2] that the irradiated metal contains stress concentrators in the form of brittle cracks which can also be caused by plastic deformation.

It is clear that such processes occur in completely different scenarios in the granular substance, of any degree of packaging. Such a substance, so to speak, is already completely brittle (fragile). Therefore, the use of micromechanical systems as protective modules those are in direct contact with radiation looks like an attractive innovative idea [3]. Of course, the most effective will be a material in which, together with disorder can be achieved (in a relatively simple way) the maximum compaction to provide simultaneously most effective screening.

In the proposed work, we will focus on the question of how to achieve maximum functional properties in a bi-component granular system, both in terms of absolute value of compaction and maximum speed of their approaching.

The study of the physical properties of simple bicomponent mixtures using the methods of statistical mechanics is one of the traditionally relevant and at the same time complex problems of statistical physics of solutions. A well-known theoretical approach in this field, which has a statistical substantiation, is the Kirkwood-Buff (KB) approach [4], which allows us to write down the basic thermodynamic quantities in terms of correlation integrals.

As it is known, granular mixtures (for example, twocomponent) show different degrees of packaging depending on the asymmetry (the ratio of molar fractions and the size of their components). Although the boundary fraction of the package can be parameterized using purely geometric constructions (VoronoiDelaunay), the dynamics of granules in conglomerations under the influence of external gentle perturbations (in particular the approaching the state which is maximally packed) is quite difficult to be described, a multiparametric process.

The method of geometric constructions of Voronoi due to its purely geometric nature, allows describing the structure of a fairly wide class of systems. To construct them for a given set of points, it is necessary to allocate a space around each point of the system, in which are all the points that are closest to it, with the translation of this condition to the other points of the system. Thus, each point (the coordinate of which is determined by the position of the center of the particle-granule) is associated with its own characteristic volume (or area).

Moreover, the volumes of the figures are additive and completely cover the volume of the system. This is an important feature for the application of the approach in the case of granular mixtures. It is important to note that 
the nature of the interaction between the particles does not directly affect the partitioning procedure [5]. Experimental studies of granular mixtures allow to obtain information about the distribution of particle centers at a certain point in time and, thus, to parameterize the structure using the Voronoi method. The study of the structure using geometric constructions of Voronoi is based on the analytical analysis of the area distributions of all particles of the system.

Obviously, this approach allows obtaining information not only about the order (symmetry) or disorder of the system, but also about the distribution of free volume and its changes that occur due to external perturbations. Free volume is the main parameter that controls the behavior of granular mixtures. It has been experimentally observed that the characteristic compaction time may anomaly increase under the conditions of approaching the ratio of the relation between particle sizes of the components to some critical value.

The mobility of the granules in the package is the lowest in the vicinity of the impregnation threshold (when small particles can still pass through the voids left between the large ones). There have also been reports of acceleration in the dynamics of compaction, with sufficiently large values of the ratio of the size of the components [6].

The question of what is the maximum number of solid identical spherical particles (granules) which can fill a given volume (a given shape) - belongs to one of the traditional mathematical problems [7-11].

A characteristic parameter that characterizes the degree of packaging is usually the volume of all particles divided by the observed volume. It is assumed that its maximum value corresponds to the face-centered symmetry, $(f c c) \eta_{f c c}=\pi / \sqrt{18}=0,74$. In this case, the characteristic scale of packaging (so-called disordered or frozen packaging, $(r c p)$ corresponds to $\eta_{r c p}=0,64$. At the same time s.c. disordered loose packaging, which characterizes the mechanically stable configuration in the Earth's gravitational field characterized by $\eta_{r l p}=0,60[12-14]$.

Under conditions of reduced gravity (micro-gravity), according to some data $[8,14,15] \eta$ may decrease till to $\eta_{r c p}=0,55$. It should be noted that the relationship between and the nature of the location of the granules in the packaged structure still remains insufficiently studied $[8,14]$.

When the degree of packaging exceeds $\eta_{f c c}$, the effects of ordering (symmetrization) of the system are observed. In particular, in [16-18] it is reported about the observation (subject to certain conditions) of the phenomenon of crystallization in the packaging of solid spheres. In two-dimensional hard disk systems, crystallization was observed during compaction under gentle shaking of the system [16].

Crystallization in a number of works is actually recognized as a driver for the processes of granular compaction, and the laws of growth of the crystalline phase quite satisfactorily describe the patterns of compaction. A three-dimensional problem is usually more complex and at the same time closer to its practical application. In some works, a scenario is assumed in which tetrahedral structures appear as growth nuclei of dense regions [18]. It should also be mentioned that crystallization can be obtained by immersing the system in an external field of vibration accelerations $[19,20]$, or by perturbations such as cyclic shift [21].

Given the data indicating that granular mixtures are packaged much denser than monodisperse (consisting of the same, in the simplest case, spherical granules) questions about the dependence of the parameters of such processes on the composition and particle size is very relevant. In this way, both direct physical [22] and numerical [23-25] studies are conducted. Both empirical [22] and numerical [23-25] studies in the above areas have been performed.

Thus, in [26] a granular mixture consisting of grains of different sizes is studied numerically. The authors came to the conclusion that the ratio of size and molar concentrations of the components of the mixture are the main parameters that determine the formation of the current and limit (maximum achievable) fractions of the package. Moreover, due to the fact that the vibration of groups of particles is a property that is reliably available for parameterization only in numerical simulations, it is a factor that actually prevents the observation of changes in the parameters of the mixture.

Sometimes [27] such difficulties are not the subject of discussion by the authors of publications, and this in fact leaves unclear the role of the above factor.

As proposed in [28], we can consider two limit cases for binary mixtures made of small and large balls. In the first case, the proportion of large (sized) particles is high, so that they form a system where smaller particles occupy the corresponding voids left by large ones. When the particle size ratio is large enough, smaller particles can propagate through the voids around the large ones.

Conversely, when the proportion of small particles increases, large particles are isolated in the environment of small ones. In the first case, the system can be conditionally classified as "gravel", while in the second, as "pudding". It is clear that both extreme cases lead to different values of the package. However, the theoretical description of such extreme cases is usually made separately from each other. A common approach containing both of the above limit cases has not yet existed.

\section{Methodology}

Our work will be devoted to the development of such an approach. In this way, we propose to use the elements of statistical physics, despite the naturally dissipative nature of the studied systems. Almost all of them have a set of s.c. quasi-stationary states in which they can be considered as approximately satisfying the criteria for using the apparatus of statistical mechanics to determine their macroscopic properties. 
The classical Kirkwood-Buff formula for a bicomponent mixture has the form [29]:

$$
\beta_{T}^{(12)}=\frac{1}{k_{B} T} \cdot \frac{1+n_{1} G_{11}+n_{2} G_{22}+n_{1} n_{2}\left(G_{11} G_{22}-G_{12}{ }^{2}\right)}{n_{1}+n_{2}+n_{1} n_{2}\left(G_{11}+G_{22}-2 G_{12}\right)},(1)
$$

where $\beta_{T}^{(12)}$ - isothermal compressibility of the mixture of two components which are numbered as 1 and 2; $n_{1}=N_{1} / V$ and $n_{2}=N_{2} / V$ - are partial densities of the number of particles, respectively; $G_{11}, G_{22}$ and $G_{12}$, are the so-called correlation integrals; $k_{B} T$ - the scale of energy.

Correlation integrals $G_{\alpha \beta}$ are related to partial structural factors $S_{\alpha \beta}(0)$ by the following relationship:

$$
S_{\alpha \beta}(0)=x_{\alpha} \delta_{\alpha \beta}+x_{\alpha} x_{\beta} n_{12} G_{\alpha \beta},
$$

where, $x_{\alpha}, x_{\beta}$ - molar fractions of the components of the mixture; $\delta_{\alpha \beta}$ - delta symbol of Kronecker; $n_{12}=N / V=\left(N_{1}+N_{2}\right) / V=n_{1}+n_{2}$ - the density of the number of particles for the mixture.

Using formula (2) it is easy to obtain:

$$
\begin{aligned}
& S_{11}(0)=(1-x)+(1-x)^{2} n_{12} G_{11}, \\
& S_{22}(0)=x+x^{2} n_{12} G_{22}, \\
& S_{12}(0)=x(1-x) n_{12} G_{12},
\end{aligned}
$$

Substituting relations (3) -(5) to the Kirkwood-Buff formula (1), we find the relationship between the isothermal compressibility of the bi-component mixture and the partial structural factors:

$$
\beta_{T}^{(12)}=\frac{1}{n_{12} k_{B} T} \cdot \frac{S_{11}(0) S_{22}(0)-S_{12}^{2}(0)}{x^{2} S_{11}(0)-2 x(1-x) S_{12}(0)+(1-x)^{2} S_{22}(0)} \text {. (6) }
$$

Due to the fact that the structural factor of a monodisperse system $S(0)$ is defined as

$$
S(0)=1+n G=n k_{B} T \beta_{T},
$$

and taking into account (3) and (4), we find expressions for partial structural factors of monodisperse phases $S_{11}(0)$ and $S_{22}(0)$ expressed in terms of partial isothermal compressibilities $\beta_{T}^{(1)}$ and $\beta_{T}^{(2)}$ :

$$
\begin{aligned}
& S_{11}(0)=(1-x) \cdot n_{1} k_{B} T \beta_{T}^{(1)}, \\
& S_{22}(0)=x \cdot n_{2} k_{B} T \beta_{T}^{(2)} .
\end{aligned}
$$

Partial isothermal compressibility can be determined using, for instance, the model Carnahan-Starling equation [30]:

$$
\beta_{T}^{(i)}=\frac{1}{n_{i} k_{B} T} \cdot \frac{\left(1-\eta_{i}\right)^{4}}{1+4 \eta_{i}+4 \eta_{i}^{2}-4 \eta_{i}^{3}+\eta_{i}^{4}},
$$

where $i=1 ; 2 ; \eta_{i}=\frac{1}{6} \pi \sigma_{i}^{3} n_{i}$ - is a parameter which characterize the degree of compaction .

Using data on the isothermal compressibility of the mixture obtained from alternative sources, for example, from experiment or by numerical simulation, and solving Eq.(6) with respect to the structure factor we obtain:

$$
\begin{gathered}
S_{12}(0)= \pm \sqrt{x^{2}(1-x)^{2} f_{12}^{2}-f_{12}\left[x^{2} S_{11}+(1-x)^{2} S_{22}\right]+S_{11} S_{22}} \\
+x(1-x) f_{12},
\end{gathered}
$$

where $f_{12}=n_{12} k_{B} T \beta_{T}^{(12)}$ is the value which in their turn can be determined on the basis of the equation of state for binary mixture of spherical particles using the formula [31]:

$$
\beta_{T}^{(12)}=\frac{1}{n_{12} k_{B} T} \cdot \frac{(1-\eta)^{4}}{1+a \eta+b \eta^{2}+c \eta^{3}+d \eta^{4}},
$$

where $a, b, c, d$ depend on $x$ (or $f=\eta_{2} / \eta$ ) and on $\alpha=\sigma_{1} / \sigma_{2}$ (where $\sigma_{1}$ and $\sigma_{2}$ the diameters of the $1^{\text {st }}$ and $2^{\text {nd }}$ components of the mixture, respectively).

Here are also useful relationships:

$$
f=\frac{x}{\alpha^{3}-\left(\alpha^{3}-1\right) \cdot x} ; \quad x=\frac{\alpha^{3} \cdot f}{1+\left(\alpha^{3}-1\right) \cdot f} \text {. }
$$

In [6], experimental data were obtained for compacting a binary mixture of spherical particles with varying degrees of size difference $\alpha=2 \div 35$. The parameterization of the obtained data is actually carried out in two characteristic limits (states) of the mixture in one of which the content of larger particles (gravel) prevails, and in the other - smaller in size (pudding). In [32], an attempt is made to describe the obtained experimental data in all intermediate states using weight functions that play a role of verifying parameters and, strictly speaking, are not cardinally different from the approach which use the separate description of compaction in the vicinities of two limit states.

Thus, despite the previous results, the theoretical description of the macroscopic properties (including compaction) of a binary mixture within a general theory still remains an actual task.

\section{Results and discussion}

In our work, has been developed a continuous approach to the description of compaction of mixture in all intermediate states using elements of statistical theory of mixtures and model equations of state. The obtained results are compared with the data of direct experimental measurements performed in [6].

Namely, taking into account formulas (3)-(4), (8)(10), and (12), let define first $S_{12}(0)$ (by use Eq.(11)). Figure 1 shows the results of the corresponding numerical calculations $S_{12}(0)$ performed using experimental data obtained in [6].

The qualitative composition of the local structure in the case of a mixture is almost impossible to establish [28]. We use a set of the values of the parameter $S_{12}(0)$, which is determined by comparing the theoretically obtained expression with their experimental observations. Along this way, using the Kirkwood-Buff formula (1), taking into account the Eq.(10) and Eq.(12) obtained on the basis of the Carnahan-Starling equations of state, we can obtain a twelfth order algebraic equation with respect to the packing parameter. Figure 2 presents the data of numerical solutions of this equation in comparison with the corresponding experimental data from [6]. 


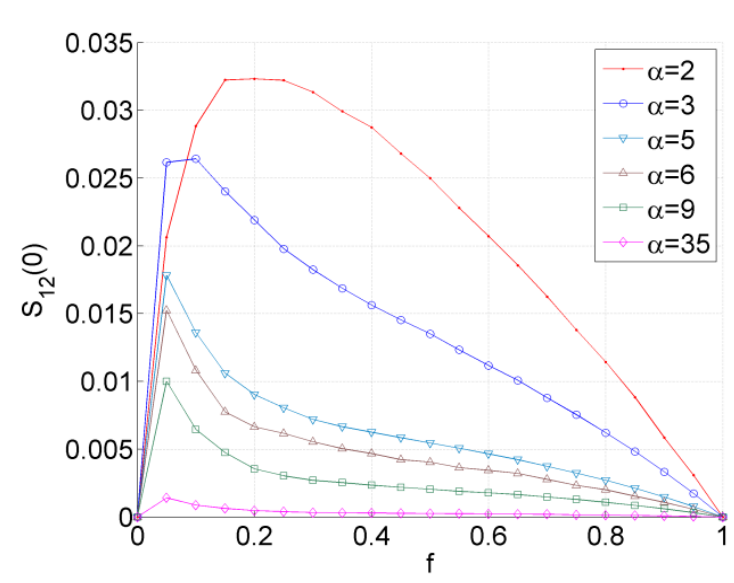

Fig. 1. Partial structure factor $S_{12}(0)$ of binary granular mixture plotted versus volume fraction of smaller component $f$. Solid line show the result of theoretical calculations which has been done with help of Eq.(11) under the different values of parameters $\alpha$.

As can be seen from Fig. 2, we obtained a good agreement of the theoretical approach with the data of experimental observations in the whole range of possible values of the volume fraction. Note that the theoretical models involved, unlike previous approaches, do not contain restrictions on the volume fraction of the components.

Another remark should be made in relation to the packing rate of the bi-component micro-mechanical mixture for which in [6], the possibility of abnormal growth is announced.

Taking into account that the packaging of the granular substance is described by a logarithmic law obtained in [19], one can write the following estimation for relaxation time $\tau \sim \exp \left(\frac{\eta \eta_{\infty}}{\eta_{\infty}-\eta}\right)$. This estimation seems to be in accordance with the prognosis for the characteristic packing time that follows from the free volume model [33] under conditions $\eta \rightarrow \eta_{\infty}$. However, it should be noted that the formula for $\tau$ is established for a monodisperse system, and strictly speaking, its adequate application to a granular mixture requires a more substantial justification, which is currently lacking.

The models involved use the notion of granular particles in the form of solid spheres, albeit with different diameters, which for real systems is, of course, idealization. The influence of particle morphology on their structural agglomeration was studied in [28]. In particular, it was found that the morphology of the granules significantly affects the dynamics of compaction. Anisotropic grains lead to two different sealing modes, separated by a "burst" of the packaging fraction. There is friction, which changes the order of placement of grains in the pile. These observations are also confirmed by numerical simulations. The possible appearance of cohesive forces between the particles can lead to the containment of the seal and reduce the values of the fraction of the package.

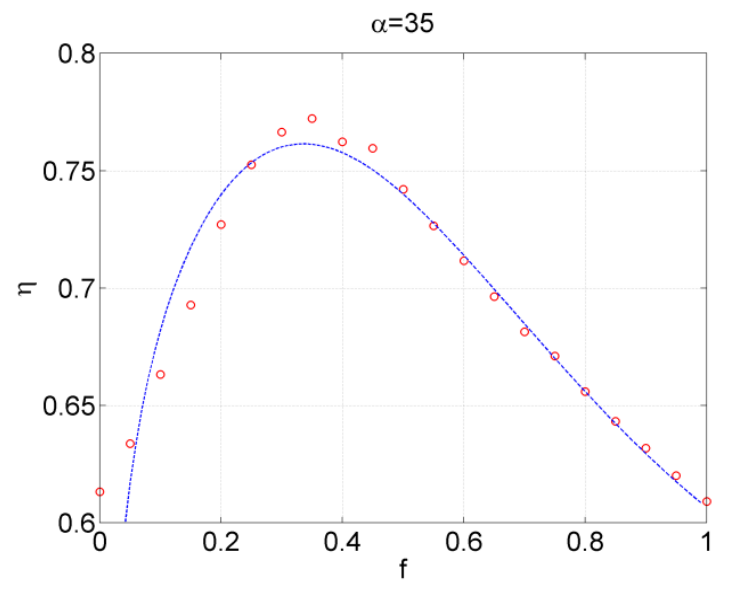

Fig. 2. Packing parameter $\eta$ for binary granular mixture plotted versus volume fraction $f$ by use Eqs.(6) and (12). Dashed line correspond to theoretical calculations; circles - to experimental data from [6].

\section{Conclusion}

Based on the analysis of experimental data we study theoretically the dynamics of compaction of the binary granular systems. We outline the impact on these processes the ratio of component sizes and partial parameters. We report also theoretically the fundamental possibility of increasing the degree and speed of packaging. In order to theoretically describe substantiate empirical data in the full range of values of the volume (or molar) fraction, it is proposed to use the apparatus of Kirkwood-Buff theory in combination with models such as Carnahan-Starling, together with a relevant phenomenological data.

Theoretical results coincide quite satisfactory with the experimental ones. The obtained data can be used to take into account the impact of multi-dispersion onto compaction properties of dry granular mixtures. These allow to build innovative approaches to optimize the manipulative dynamics of compaction of discrete micromechanical materials to control the ability of granular mixture to change local structure, degree and rate of packing and also some other parameters. All said vide supra permit to provide a necessary optimization of a relevant safety technologies which use a granular components.

\section{References}

1. T. Uchida, Y. Kawahara, Y. Hayashi, A. Tateishi, J. Hydraul. Eng. 146, 04020071 (2020)

2. J. F. Knott, Fundamentals of fracture mechanics (Metallurgy, Moscow, 1978) [in Russian]

3. O. I. Gerasymov, M. M. Khudyntsev, A. Y. Spivak, I. S. Andrianova, Granular materials in utilization technologies of radiation-harmful substations, in Proceedings of the National Forum "Problems, perspectives and strategy of waste utilization in Ukraine", 22-23 November 2016, Kyiv, Ukraine (2016) [in Ukrainian] 
4. O. I. Gerasymov, M. M. Somov, Ukr. J. Phys. 60, 324 (2015)

5. O. I. Gerasymov, A. G. Zagorodny, M. M. Somov, Ukr. J. Phys. 58, 32 (2013)

6. S. Pillitteri, G. Lumay, E. Opsomer, N. Vandewalle, Sci. Rep. 9, 7281 (2019)

7. H. M. Jaeger, S. R. Nagel, Science 225, 1523 (1992)

8. T.Aste, D. Weaire, The Pursuit of Perfect Packing (CRC Press, 2000)

9. D. A. Weitz, Science 303, 968 (2004).

10. S. Torquato, J. Chem. Phys. 149, 020901 (2018).

11. S. Torquato, F. H. Stillinger, Rev. Mod. Phys. 82, 2633 (2010)

12. J. G. Berryman, Phys. Rev. A 27, 1053 (1983).

13. G. Y. Onoda, E. G. Liniger, Phys. Rev. Lett. 64, 2727 (1990)

14. C. Song, P. Wang, H. A. Makse, Nature 453, 629 (2008)

15. M. Noirhomme, F. Ludewig, N. Vandewalle, E. Opsomer, Phys. Rev. E 95, 022905 (2017)

16. G. Lumay, N. Vandewalle, Phys. Rev. Lett. 95, $028002(2005)$

17. O. Carvente, J. Ruiz-Suarez, Phys. Rev. Lett. 95, 018001 (2005)

18. A. Panaitescu, K. A. Reddy, A. Kudrolli, Phys. Rev. Lett. 108, 108001 (2012)

19. J. B. Knight, C. G. Fandrich, C. N. Lau, H. M. Jaeger, S. R. Nagel, Phys. Rev. E 51, 3957 (1995)

20. S. K. de Richter, C. Hanotin, P. Marchal, S. Leclerc, F. Demeurie, N. Louvet, Eur. Phys. J. E 38, 74 (2015)

21. M. Nicolas, P. Duru, O. Pouliquen, Eur. Phys. J. E 3, 309 (2000)

22. G. Roquier, Powder Technol. 302, 247 (2016)

23. R. S. Farr, R. D. Groot, J. Chem. Phys. 131, 244104 (2009)

24. M. Danisch, Y. Jin, H. A Makse, Phys. Rev. E 81, 051303 (2010)

25. D. Chen, S. Torquato, Phys. Rev. E 92, 062207 (2015)

26. A. B. Hopkins, Y. Jiao, F. H. Stillinger, S. Torquato, Phys. Rev. Lett. 107, 125501 (2011)

27. R. P. Behringer, B. Chakraborty, Rep. Prog. Phys. 82, 012601 (2018)

28. T. Boutreux, P. G. de Gennes, Physica A 244, 59 (1997)

29. J. G. Kirkwood, F. P. Buff, J. Chem. Phys. 19, 6, 774 (1951)

30. N. F. Carnahan, K. E. Starling, J. Chem. Phys. 51, 2, 635 (1969)

31. G. A. Mansoori, N. F. Carnahan, K. E. Starling, T. W. Leland jr., J. Chem. Phys. 54, 1523 (1971)

32. S. Pillitteri, E. Opsomer, G. Lumay, N. Vandewalle, Soft Matter 16, 39, 9094 (2020)
33. O. I. Gerasymov, N. N. Khudyntsev, O. A. Klymenkov, A. Y. Spivak, Ukr. J. Phys. 50, 623 (2005) 